\title{
Effects of An Acute Increase in Atrial Pressure on Atrial Refractoriness in Humans
}

\author{
HUGH CALKINS, RAFEL EL-ATASSI, STEVEN KALBFLEISCH, \\ JONATHAN LANGBERG, and FRED MORADY
}

From the Division of Cardiology, University of Michigan Medical Center, Ann Arbor, Michigan

\begin{abstract}
CALKINS, H., ET AL.: Effects of An Acute Increase in Atrial Pressure on Atrial Refractoriness in Humans. Contraction-excitation feedback has been studied extensively in mammalian ventricles. In contrast, little is known about contraction-excitation feedback in mammalian atria. The objective of this study was to investigate the effect of acute alterations in atrial pressure, induced by varying the atrioventricular (AV) interval, on atrial refractoriness. Twenty patients without structural heart disease participated in the study. In each patient the atrial effective (ERP) and absolute refractory periods (ARP) were measured during $A V$ pacing at a cycle length of $500 \mathrm{msec}$ and an AV interval of $120 \mathrm{msec}$. Acute increases in atrial pressure were induced by pacing the atrium and ventricle simultaneously for the final two beats of the drive train. The ERP was defined as the longest extrastimulus coupling interval that failed to capture with an extrastimulus current strength of twice the stimulation threshold. The ARP was defined in a similar manner with an extrastimulus current strength of $10 \mathrm{~mA}$. The ERP and ARP were determined using the incremental extrastimulus technique. A subset of patients had the pacing protocol performed during autonomic blockade. As the AV interval of the final two beats of the drive train was shortened from $120 \mathrm{msec}$ to $0 \mathrm{msec}$, the peak right atrial pressure increased from $7 \pm 3 \mathrm{mmHg}$ to $15 \pm 5 \mathrm{mmHg}(P$ $<0.001)$. The increase in atrial pressure associated with simultaneous pacing of the atrium and ventricle resulted in shortening of the atrial ERP and ARP by $7.3 \pm 5.2$ and $6.2 \pm 3.5 \mathrm{msec}$, respectively $(\mathrm{P}<$ 0.001). Similar results were obtained during autonomic blockade. These findings confirm the presence of contraction-excitation feedback in normal human atria. (PACE, Vol. 15, November, Part I 1992)
\end{abstract}

contraction-excitation feedback, refractoriness, atrioventricular pacing, atrioventricular interval

\section{Introduction}

Contraction-excitation feedback, the phenomenon whereby changes in mechanics of myocardial contraction precede and result in changes in membrane potential, has been studied extensively in mammalian ventricles. ${ }^{1-10}$ These studies have confirmed the presence of contraction-excitation feedback and have also demonstrated the importance of the timing of load alterations in affecting electrophysiological phenomena. In contrast, few studies have investigated contraction-excitation

Address for reprints: Hugh Calkins, M.D., Johns Hopkins Hospital, Electrophysiology, Carnegie-Room 530, 601 Wolfe Street, Baltimore, MD 21287.

Received April 2, 1992; revision May 20, 1992; accepted May $30,1992$. feedback in mammalian atria. ${ }^{11-13}$ The results of these studies conflict with each other and/or with the results of prior studies in mammalian ventricles. ${ }^{1-6}$ Therefore, it is uncertain whether the phenomenon of contraction-excitation feedback exists in the human atrium.

The purpose of this study was to investigate the phenomenon of contraction-excitation feedback in the human atrium by studying the influence of acute changes in atrial pressure on atrial refractoriness. Atrial pressure was acutely increased by pacing the atrium and ventricle simultaneously for two beats prior to measurement of refractoriness. The effects of an acute increase in atrial pressure on the effective (ERP) and absolute refractory periods (ARP) were evaluated in the baseline state and also during autonomic blockade. 


\section{Methods}

\section{Subjects Studied}

The subjects of this study were 20 patients without underlying structural heart disease who underwent a clinically-indicated electrophysiological test. Seventeen patients had previously undergone radiofrequency catheter ablation of an accessory atrioventricular (AV) connection or modification of the AV node, and the study protocol was performed during a follow-up electrophysiological test. The electrophysiological test in the remaining three patients was performed for evaluation of syncope or paroxysmal supraventricular tachycardia. None of the patients had a history of atrial fibrillation. There were 13 women and 7 men. Their mean age was $36 \pm 17$ years ( \pm standard deviation). Three additional patients were enrolled in the protocol but were excluded because of the inability to complete the protocol due to the development of sustained atrial fibrillation.

\section{Electrophysiological Testing}

Electrophysiological tests were performed in the fasting, unsedated state at least five half-lives after discontinuation of antiarrhythmic drug therapy including beta blockers and calcium channel blockers. Informed consent was obtained under an investigational protocol approved by the Human Research Committee at the University of Michigan. Three quadripolar electrode catheters were inserted into a femoral vein and positioned in the high right atrium, His-bundle position, and apex of the right ventricle. After completion of the clinically-indicated portion of the electrophysiological test, the electrode catheter in the His-bundle position was replaced with a 6 French multipurpose central lumen catheter, which was positioned in the mid-right atrium for recording atrial pressure. Leads VI, I, and III, the intracardiac electrograms, and the right atrial pressure were recorded on a Siemens-Elema (Solna, Sweden) Mingograf 7 recorder.

\section{Pacing Protocol}

Pacing was performed using a programmable stimulator (Bloom Associates, Reading, PA, USA) with pulses $2 \mathrm{msec}$ in duration and a current inten- sity twice the stimulation threshold. The atrial stimulation threshold was $<1.5 \mathrm{~mA}$ in each subject and the mean stimulation threshold was 0.8 $\pm 0.3 \mathrm{~mA}$. The ARP and ERP of the right atrium were determined using an AV pacing drive cycle length of $500 \mathrm{msec}$, a drive train duration of 12 beats, and an intertrain pause of 1 second. The AV interval during the basic drive train was $120 \mathrm{msec}$. In order to maximize reproducibility of the measurements of refractoriness, the initial beat of the drive train was synchronized to occur $500 \mathrm{msec}$ after a sinus beat and the determinations of the atrial ARP and ERP were preceded by a 3-minute conditioning period during which time basic trains were introduced without the extrastimulus. ${ }^{14}$ An atrial extrastimulus was introduced after every 12 th atrial paced beat at a coupling interval shorter than the estimated refractory period (typically $160 \mathrm{msec}$ ). The coupling interval of the extrastimulus was increased in steps of $2 \mathrm{msec}$ until atrial capture occurred. The ERP was defined as the longest extrastimulus coupling interval that failed to capture the atrium at an extrastimulus current strength of twice the stimulation threshold. The ARP was measured in a similar manner using a drive train current strength of twice diastolic threshold and an extrastimulus current strength of $10 \mathrm{~mA}$.

The ERP and ARP were determined in this fashion in 12 patients, then were measured again following an acute increase in atrial pressure. Acute increases in atrial pressure were induced by shortening the AV relationship of the final two beats of the 12-beat drive train from $120 \mathrm{msec}$ to 0 msec. Finally, the atrial ERP and ARP were measured again at an AV interval of 120 msec to evaluate reproducibility. If atrial fibrillation was induced during determinations of the ERP or ARP, pacing was discontinued until sinus rhythm returned. Data were considered reproducible and acceptable for analysis only if the first and final determinations of the ERP and ARP at an AV interval of $120 \mathrm{msec}$ did not differ by more than $4 \mathrm{msec}$. If the reproducibility criterion was not met, the measurements were repeated. The ERP was measured because it is used conventionally to measure refractoriness during clinical electrophysiological tests. The ARP was measured because prior studies have demonstrated that the largest effect of 
load on refractoriness is detected at high stimulus intensities. ${ }^{2,3}$

\section{Hemodynamic Measurements}

The peak and mean right atrial pressures were recorded during pacing with an AV interval of 120 msec. The peak right atrial pressure of the last beat of the drive train was recorded immediately prior to measurements of refractoriness at each AV interval.

\section{Autonomic Blockade}

To eliminate the possible effects of fluctuations in autonomic tone, the pacing protocol was performed in a second group of eight patients following autonomic blockade. Autonomic blockade was achieved by administration of $0.04 \mathrm{mg} / \mathrm{kg}$ of atropine and $0.2 \mathrm{mg} / \mathrm{kg}$ of propranolol intravenously over a 10-minute period. The mean dose of propranolol was $13.3 \pm 1.9 \mathrm{mg}$ and the mean dose of atropine was $2.6 \pm 0.3 \mathrm{mg}$. Previous studies have demonstrated that these amounts of propranolol and atropine result in complete autonomic blockade. ${ }^{16}$ To confirm that the effect of these agents was constant for the duration of the pacing protocol, the sinus rate was measured immediately before and after the pacing protocol was completed. The sinus cycle length immediately following administration of propranolol and atropine but prior to the pacing protocol was no different than at the completion of the pacing protocol (656 $\pm 51 \mathrm{msec}$ vs $671 \pm 59 \mathrm{msec} ; \mathrm{P}=0.3$ ).

\section{Statistical Analysis}

All data are expressed as mean \pm 1 standard deviation. A repeated measures analysis of variance was used to evaluate changes in refractoriness and pressure at differing AV intervals. Differences in the change in refractoriness in the baseline state versus under denervated conditions were compared using the Student's t-test. A P value of 0.05 or less was considered significant.

\section{Results}

\section{Baseline Findings}

Table I describes the effects of altering the AV interval on the peak right atrial pressure, atrial ERP, and atrial ARP under baseline conditions. The mean right atrial pressure was $3 \pm 2 \mathrm{mmHg}$ during the basic drive train with an AV interval of 120 msec. When the AV interval of the final two beats of the basic drive train was decreased from $120 \mathrm{msec}$ to $0 \mathrm{msec}$, the peak right atrial pressure increased from $7 \pm 3 \mathrm{mmHg}$ to $15 \pm 5 \mathrm{mmHg}$ (P $<0.001)$. The atrial ERP measured following two beats of simultaneous pacing of the atrium and ventricle was $7.3 \pm 5.2 \mathrm{msec}$ (median $=8$, range $0-18, \mathrm{P}<0.001$ ) shorter than that measured with an AV interval of $120 \mathrm{msec}$ (Fig. 1). The final atrial ERP, measured with an AV interval of $120 \mathrm{msec}$ to confirm reproducibility, did not differ from the initial measurement of the ERP with an AV interval of 120 msec.

The atrial ARP measured following two beats

Table I.

Relationship Between AV Interval, Atrial Pressure, and Atrial Refractoriness

\begin{tabular}{lcccc}
\hline & \multicolumn{3}{c}{ AV Interval } & \\
\cline { 2 - 4 } & $\mathbf{1 2 0} \mathbf{~ m s e c}$ & $\mathbf{0 ~} \mathbf{~ m s e c}$ & $\mathbf{1 2 0} \mathbf{~ m s e c}$ & P \\
\hline ERP (msec) & $208 \pm 19$ & $199 \pm 21$ & $208 \pm 19$ & $<0.001$ \\
ARP (msec) & $180 \pm 17$ & $171 \pm 16$ & $179 \pm 17$ & $<0.001$ \\
RA peak pressure (mmHg) & $7 \pm 3$ & $15 \pm 5$ & $7 \pm 3$ & $<0.001$ \\
\hline
\end{tabular}

Values are expressed as mean \pm standard deviation. AV interval $=$ interval between the atrial and ventricular stimulus in the final two beats of the basic drive train; ARP = absolute refractory period; ERP = effective refractory period; RA peak $=$ peak right atrial pressure. 

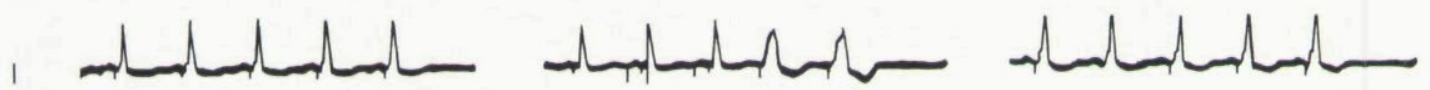

RA
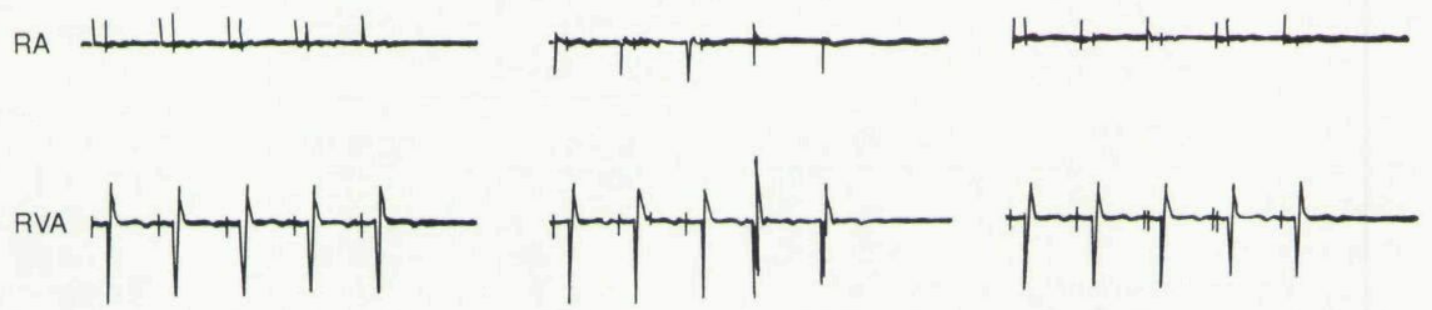

$\begin{array}{ll}\operatorname{ERP}(\mathrm{msec}) & 234 \\ \operatorname{ARP}(\mathrm{msec}) & 206\end{array}$
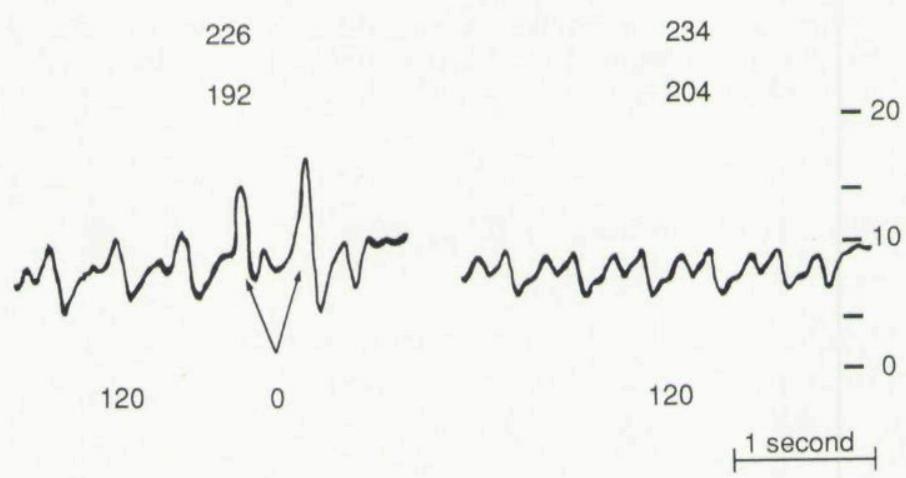

Figure 1. Recording of right atrial pressure during AV pacing with a drive train AV interval of $120 \mathrm{msec}$ and varying AV intervals of the final two beats of the drive train. Shown are surface lead I, and intracardiac electrograms recorded from the right atrium (RA), right ventricular apex (RVA), and the right atrial pressure. The final five beats of the drive train are shown. In the first and third panel, the AV interval is fixed at $120 \mathrm{msec}$. In the middle panel, the AV interval is 0 msec for the final two beats of the drive train. This shortening of the AV interval results in a marked increase in the peak atrial pressure. Also shown are the atrial effective refractory period (ERP) and absolute refractory period (ARP) that were measured during pacing with each $A V$ interval.

of simultaneous pacing of the atrium and ventricle was $9.5 \pm 5.8$ msec $($ median $=10$, range $0-18$ msec, $\mathrm{P}<0.001$ ) shorter than that measured with an AV interval of 120 msec. The final atrial ARP, measured with an AV interval of $120 \mathrm{msec}$ to confirm reproducibility, did not differ from the initial measurement of the ARP with an AV interval of 120 msec.

\section{Autonomic Blockade}

The effects of alterations in the AV interval on the peak right atrial pressure and on the atrial ERP and ARP following pharmacological autonomic blockade are shown in Table II. When the AV interval of the final two beats of the basic drive train decreased from $120 \mathrm{msec}$ to $0 \mathrm{msec}$, the peak right atrial pressure increased from $8 \pm 3 \mathrm{mmHg}$ to 15 $\pm 5 \mathrm{mmHg}(\mathrm{P}<0.001)$. The atrial ERP measured following two beats of simultaneous pacing of the atrium and ventricle was $4 \pm 3.7 \mathrm{msec}$ (median $=$ 4 , range -2 to $10, \mathrm{P}<0.001$ ) shorter than that measured with an AV interval of $120 \mathrm{msec}$. The atrial ARP measured following two beats of simultaneous pacing of the atrium and ventricle was 6.2 \pm 3.5 msec $($ median $=6$, range $0-12 \mathrm{msec}, \mathrm{P}<$ 0.001 ) shorter than that measured with an AV interval of 120 msec. The changes in ERP and ARP and the change in peak right atrial pressure induced by shortening of the AV interval were no different than in the baseline state $(\mathrm{P}>0.1)$. 
Table II.

Relationship Between AV Interval, Atrial Pressure, and Refractoriness Following Autonomic Blockade

\begin{tabular}{lcccc} 
& \multicolumn{3}{c}{ AV Interval } & \\
\cline { 2 - 4 } & $\mathbf{1 2 0} \mathbf{~ m s e c}$ & $\mathbf{0 ~} \mathbf{~ m s e c}$ & $\mathbf{1 2 0} \mathbf{~ m s e c}$ & $\mathbf{P}$ \\
\hline ERP (msec) & $230 \pm 24$ & $226 \pm 26$ & $229 \pm 24$ & 0.02 \\
ARP (msec) & $202 \pm 20$ & $196 \pm 20$ & $201 \pm 20$ & $<0.001$ \\
RA peak pressure $(\mathrm{mmHg})$ & $8 \pm 3$ & $15 \pm 3$ & $9 \pm 3$ & $<0.001$ \\
\hline
\end{tabular}

Values are expressed as mean \pm standard deviation. AV interval $=$ interval between the atrial and ventricular stimulus in the final two beats of the basic drive train; ARP = absolute refractory period; ERP = effective refractory period; RA peak = peak right atrial pressure.

\section{Effect of the Site of Ventricular Activation During the Basic Drive Train}

During the basic drive train, a variable degree of ventricular fusion was observed between patients at an AV interval of $120 \mathrm{msec}$. Figure 1 shows ventricular activation occurring primarily via the AV node during the basic drive train. In other patients, minimal fusion was observed during the basic drive train with ventricular activation originating almost entirely from the site of ventricular pacing at the apex of the right ventricle. To evaluate whether the site of ventricular activation influenced the effect of an acute shortening of the AV interval on atrial refractoriness, we compared patients in whom ventricular activation occurred primarily via the AV node (7 patients) with those patients in whom ventricular activation originated primarily from the pacing site (13 patients). In patients in whom conduction occurred primarily via the AV node, the atrial ERP shortened from $223 \pm 24 \mathrm{msec}$ to $217 \pm 27 \mathrm{msec}(\mathrm{P}=$ 0.001), and the atrial ARP shortened from $181 \pm$ $19 \mathrm{msec}$ to $171 \pm 17 \mathrm{msec}(\mathrm{P}<0.001)$ as the AV interval was deceased from $120 \mathrm{msec}$ to 0 msec. Similarly, in the subgroup of patients in whom ventricular activation occurred primarily from the pacing site, the atrial ERP shortened from $205 \pm$ $20 \mathrm{msec}$ to $197 \pm 22 \mathrm{msec}(\mathrm{P}=0.02)$ and the atrial ARP shortened from $191 \pm 22$ to $183 \pm 24 \mathrm{msec}$ $(\mathrm{P}<0.001)$ as the AV interval was decreased from $120 \mathrm{msec}$ to $0 \mathrm{msec}$. The degree of shortening of the atrial ERP and the atrial ARP was similar in the two groups $(\mathrm{P}>0.1)$.

\section{Discussion}

The major finding of this study is that an acute increase in atrial pressure results in shortening of atrial refractoriness. This finding demonstrates that contraction-excitation feedback is present in the human atrium.

\section{Effects of an Acute Increase in Atrial Pressure on Refractoriness}

The results of this study demonstrate that an acute increase in right atrial pressure, induced on a beat-by-beat basis by pacing the atrium and ventricle simultaneously, shortens the atrial ERP and ARP. This finding is consistent with the results of prior experimental studies that demonstrated that acute increases in load applied during the action potential or during systole shorten repolarization. Craelius et al., ${ }^{17}$ for example, characterized stretch sensitive channels in isolated rat myocytes. Activation of these channels by stretch applied during the action potential plateau would result in a repolarizing current. Similarly, in isolated tissue, previous studies ${ }^{18}$ have demonstrated that stretch imposed immediately after the onset of the action potential shortens the duration of the action potential. Acute occlusion of the aorta or pulmonary artery in the in situ heart or an acute change from ejecting to isovolumic contraction in the isolated heart has also been shown to shorten repolarization as indexed by the duration of the monophasic action potential (MAP) recording. ${ }^{5-7}$ 


\section{Autonomic Blockade}

To exclude the possible confounding effects of alterations in sympathetic and vagal tone during pacing at varying AV intervals, the pacing protocol was performed in a second group of eight patients during pharmacological autonomic blockade. The atrial ERP and ARP shortened by a similar degree after simultaneous atrial and ventricular pacing. This provides evidence that the shortening in atrial refractoriness that accompanies an acute rise in atrial pressure is not mediated by a change in sympathetic or vagal tone. These findings are consistent with those of previous experimental studies of contraction-excitation coupling that have demonstrated that the effects of alterations in load are maintained following autonomic blockade. ${ }^{5,8}$

\section{Abrupt Versus Gradual Changes in Load}

No prior study has evaluated that effect of acute changes in atrial pressure on atrial refractoriness. However, a recent study reported the effects on refractoriness of alterations in atrial pressure induced by varying the AV interval under steadystate conditions. ${ }^{12}$ A persistent increase in atrial pressure similar to that achieved transiently in this study resulted in no change in the atrial ERP and ARP. The disparate findings of these two studies are consistent with the current understanding of contraction-excitation feedback and confirm the importance of the time course of load change. In isolated tissues, for example, several studies have shown that gradual changes in load have little effect on the action potential. ${ }^{19-21}$ In contrast, abrupt stretch imposed for a single beat immediately following onset of depolarization results in short-

\section{References}

1. Hanson DE, Craig CS, Hondeghem LM. Stretch-induced arrhythmias in the isolated canine ventricle. Circulation 1990; 81:1094-1105.

2. Calkins H, Maughan WL, Weisman HF, et al. Effect of acute volume load on refractoriness and arrhythmia development in isolated, chronically infarcted canine hearts. Circulation 1989; 79:687-697.

3. Lerman BB, Burkhoff D, Yue DT, et al. Mechanoelectrical feedback: Independent role of preload and contractility in modulation of canine ventricular excitability. J Clin Invest 1985; 76:1843-1850.

4. Calkins H, Maughan WL, Kass DA, et al. Electrophysiological effect of volume load in isolated canine hearts. Am J Physiol 1989; 25:H1697-H1706. ening of the action potential duration. ${ }^{18}$ The time course of load change has also been shown to be important in intact ventricles. Under steady-state conditions, $>100 \%$ increases in ventricular volume have been shown to result in only slight $(<$ $1 \%$ ) shortening of refractoriness and no change in repolarization as indexed by MAP recordings. ${ }^{1,4}$ In contrast, a sudden increase in load induced by acute occlusion of the aorta shortens the duration of repolarization as indexed by MAP recordings. ${ }^{5}$

\section{Limitations}

A limitation of this study is that noninvasive determination of right atrial volumes was technically not possible and, therefore, the change in right atrial wall stress could not be determined. Although atrial size was not measured in this study, previous studies have demonstrated that the left atrial size increases as the AV interval during pacing is decreased from $160 \mathrm{msec}$ to $0 \mathrm{msec}^{11}$ From this observation, we presume that right atrial size increased as the AV interval was shortened and the right atrial pressure increased.

\section{Conclusions}

The results of this study demonstrate that abrupt increases in atrial pressure induced on a beatby-beat basis shorten refractoriness. These findings confirm the presence of contraction-excitation feedback in the normal human atrium. The effect of acute changes in atrial pressure on refractoriness in structurally abnormal atria and the clinical importance of contraction-excitation feedback in the development of atrial arrhythmias remain to be determined.

5. Calkins H, Levin JH, Kass DA. Electrophysiological effect of varied rate and extent of acute in vivo left ventricular load increase. Cardiovasc Res 1991; 25: 637-644.

6. Levine JH, Guarnieri T, White RI, et al. Changes in myocardial repolarization in patients undergoing balloon valvuloplasty for congenital pulmonary stenosis: Evidence for contraction-excitation feedback in human. Circulation 1988; 77:70-77.

7. Franz MR, Burkhoff D, Yue DT, et al. Mechanically induced action potential changes and arrhythmia in isolated and in situ canine hearts. Cardiovasc Res 1989; 23:213-223.

8. Gornick CC, Tobler HG, Tuna IC, et al. Electrophys- 
iologic effects of left ventricular free wall traction in intact hearts. Am J Physiol 1989; 257(4 Pt 2): H1211-H1219.

9. Taggart P, Sutton PMI, Treasure T, et al. Monophasic action potentials at discontinuation of cardiopulmonary bypass: Evidence for contraction-excitation feedback in man. Circulation 1988; 77 1266-1275.

10. Covell JW, Lab MJ, Pavelec R. Mechanical induction of paired action potentials in intact heart in situ. J Physiol (Lond) 1981; 320:34.

11. Klein LS, Miles WM, Zipes DP. Effect of atrioventricular interval during pacing or reciprocating tachycardia on atrial size, pressure, and refractory period. Contraction-excitation feedback in human atrium. Circulation 1990; 82:60-68.

12. Calkins H, El-Atassi R, Leon A, et al. Effect of the atrioventricular relationship on atrial refractoriness in humans. PACE 1992; 15:771-778.

13. Kaseda S, Zipes DP. Contraction-excitation feedback in the atria: A cause of changes in refractoriness. J Am Coll Cardiol 1988; 11:1327-1336.

14. Kadish A, Toivonen L, Kushner J, et al. Factors that influence the reproducibility of ventricular refractory period measured by the extrastimulus technique. PACE 1990; 13:405-409.
15. Prystowsky EM, Jackman WM, Rinkenberger J, et al. Effect of autonomic blockade on ventricular refractoriness and atrioventricular nodal conduction in humans. Circ Res 1981; 49:511.

16. Reference deleted in revision.

17. Craelius W, Chen V, El-Sherif N. Stretch activated ion channels in ventricular myocytes. Biosci Rep 1988; 8:407-414.

18. Lab MJ. Transient depolarisation and action potential alterations following mechanical changes in isolated myocardium. Cardiovasc Res 1980; 14 : 624-637.

19. Sanders R, Myerburg RJ, Gelband H, et al. Dissimilar length-tension relations of canine ventricular muscle and false tendon: Electrophysiologic alterations accompanying deformation. J Mol Cell Cardiol 1979; 11:209-219.

20. Dominquez G, Fozzard HA. Effect of stretch on conduction velocity and cable properties of cardiac Purkinje fibers. Am J Physiol 1979; 237:C119C124.

21. Hennekes R, Kaufman R, Lab M, et al. Feedback loops involved in cardiac excitation-contraction coupling: Evidence for two different pathways. J Mol Cell Cardiol 1977; 9:699-713. 
This document is a scanned copy of a printed document. No warranty is given about the accuracy of the copy. Users should refer to the original published version of the material. 